\title{
The effect of a planet on the dust distribution in a 3D protoplanetary disk
}

\author{
L. Fouchet ${ }^{1,2}$, S. T. Maddison ${ }^{3}$, J.-F. Gonzalez ${ }^{1}$, and J. R. Murray ${ }^{3}$ \\ 1 Université de Lyon, Lyon 69003, Université Lyon 1, Villeurbanne 69622, CNRS, UMR 5574, Centre de Recherche Astrophysique \\ de Lyon, École Normale Supérieure de Lyon, 46 allée d'Italie, 69364 Lyon Cedex 07, France \\ e-mail: fouchet@phys.ethz.ch; Jean-Francois.Gonzalez@ens-lyon.fr \\ 2 Department of Physics, ETH Zurich, 8093 Zurich, Switzerland \\ ${ }^{3}$ Centre for Astrophysics and Supercomputing, Swinburne University of Technology, PO Box 218, Hawthorn, VIC 3122, Australia \\ e-mail: smaddison@swin.edu. au; jmurray@astro.swin.edu.au
}

Received 2 April 2007 / Accepted 28 August 2007

ABSTRACT

\begin{abstract}
Aims. We investigate the behaviour of dust in protoplanetary disks under the action of gas drag in the presence of a planet. Our goal is twofold: to determine the spatial distribution of dust depending on grain size and planet mass, and therefore to provide a framework for interpretation of coming observations and future studies of planetesimal growth.

Methods. We numerically model the evolution of dust in a protoplanetary disk using a two-fluid (gas + dust) Smoothed Particle Hydrodynamics (SPH) code, which is non-self-gravitating and locally isothermal. The code follows the three dimensional distribution of dust in a protoplanetary disk as it interacts with the gas via aerodynamic drag. In this work, we present the evolution of a minimum mass solar nebula (MMSN) disk comprising $1 \%$ dust by mass in the presence of an embedded planet. We run a series of simulations which vary the grain size and planetary mass to see how they affect the resulting disk structure.

Results. We find that gap formation is much more rapid and striking in the dust layer than in the gaseous disk and that a system with a given stellar, disk and planetary mass will have a completely different appearance depending on the grain size. For low mass planets in our MMSN disk, a gap can open in the dust disk while not in the gas disk. We also note that dust accumulates at the external edge of the planetary gap and speculate that the presence of a planet in the disk may enhance the formation of a second planet by facilitating the growth of planetesimals in this high density region.
\end{abstract}

Key words. planetary systems: protoplanetary disks - hydrodynamics - methods: numerical

\section{Introduction}

The effect of a planet in a gaseous disk has been well studied both analytically and numerically (Goldreich \& Tremaine 1979, 1980; Papaloizou \& Lin 1984; Lin \& Papaloizou 1986; Lin \& Papaloizou 1993; Kley 1999; Bryden et al. 1999; de Val-Borro et al. 2006). Planetary gaps result from tidal interactions between a sufficiently massive planet and the disk if the tidal torques induced by the planet exceed the viscous torques of the disk. If the gap is sustained, its presence severely decreases accretion of disk material by the planet (Artymowicz \& Lubow 1996; Kley 1999; Bryden et al. 1999), which suggests that the planet formation process is self-limiting. Lufkin et al. (2004) suggest, however, that the accretion rate is not so dramatically reduced in selfgravitating disks. The exchange of angular momentum that results from the tidal torques leads to planetary migration, which is slowed once a gap has formed. This is known as type II migration and proceeds on a viscous timescale (Papaloizou \& Lin 1984; Takeuchi et al. 1996; Ward 1997). If the planet mass is not massive enough to sustain a gap, it will undergo the faster type I migration (Goldreich \& Tremaine 1979; Ward 1997), while intermediate-mass planets only open a partial gap and suffer type III, or runaway, migration (Masset \& Papaloizou 2003; Artymowicz 2004).

There are a variety of observational signature of planetary gaps, including infrared dips and direct scattered light and submillimetre observations of protoplanetary disks. Mid-infrared dips are seen in quite a few pre-main sequence disks around
T Tauri stars including T Tau and GM Aur. The mid-IR emission comes from the inner 1-10 AU of the disk and the $10 \mu \mathrm{m}$ feature is predominantly from silicate grain emission and it has been suggested that such IR dips may be the results of an unseen planet clearing out the disk material (e.g. Calvet et al. 2002; Rice et al. 2003; Varnière et al. 2006). Such dips, however, have also been modelled assuming a continuous disk with a more realistic density distribution than a standard power-law (Boss \& Yorke 1996), and by a continuous disk with silicate grain growth (Dullemond \& Dominik 2005). Sub-millimetre observations of older debris disks show brightness asymmetries and a range of structures within the disk, many of which can be modelled by the presence of an unseen planet locking the dust in resonances (Ozernoy et al. 2000; Wilner et al. 2002; Deller \& Maddison 2005). However it has been noted by Wyatt (2006) and Grigorieva et al. (2007) that holes and clumpiness of debris disks can result purely from collisional cascade of planetesimals within the disk. Takeuchi \& Artymowicz (2001) show that gas drag alone can be used to explain annulus-like disks with sharp edges, and sub-mm holes have also been explained by the sublimation of icy grains (Jura et al. 1998). Direct imaging of scattered light from protostellar and debris disks with HST confirm the presence of gaps (Weinberger et al. 1999; Schneider et al. 1999; Ardila et al. 2004; Kalas et al. 2005; Schneider et al. 2006)

Recent models have indicated that ALMA will be able to detect planetary gaps at sub-millimetre wavelengths. Using the results of $2 \mathrm{D}$ hydrodynamics simulations, 
Wolf \& D'Angelo (2005) use a Monte Carlo radiative transfer code to predict the emission of a disk hosting a planetary gap carved out by a range of planet masses. They find that ALMA will be able to detect the gap in a $0.01 M_{\odot}$ disk with an $M_{\text {Jup }}$ planet at a distance of $100 \mathrm{pc}$. When using $2 \mathrm{D}$ hydrodynamics codes, one needs to add an analytic disk atmosphere in order to provide the Monte Carlo simulation with a 3D density structure. Wolf \& D'Angelo (2005) assume an un-flared disk, while Varnière et al. (2006) use a flared disk and show that the spectral energy distribution and synthetic scattered light images are effected by the outer wall of the gap. Both groups, however, assume that the gas and dust are well-mixed within the disk and yet the gas-to-dust ratio will change substantially in protoplanetary disks as the grains begin to grow and settle to the midplane (Maddison et al. 2003).

In recent years, a growing number of studies started to consider the separate evolution of gas and dust in disks. Barrière-Fouchet et al. (2005, hereafter BF05) described the simultaneous radial migration and vertical settling of dust grains, treated as a separate fluid in global 3D SPH simulations, and their dependency on grain size. Rice et al. (2004) showed that large solid bodies, treated as trace particles, tend to accumulate in the spiral arms in massive disks. The influence of the magnetorotational instability (MRI, Balbus \& Hawley 1991) has also been studied in Eulerian global 2D simulations (Fromang \& Nelson 2005) and local 3D simulations (Fromang \& Papaloizou 2006; Johansen \& Klahr 2005; Johansen et al. 2006). A fraction of the solid bodies, treated as a separate fluid for small sizes or as particles for large sizes, were found to be trapped in density maxima generated by the MHD turbulence.

As well as the gas-to-dust ratio varying throughout the disk, we expect that the effects of planetary gaps will be stronger in the dust phase than in the gas phase, which will further affect observations. Any gap created by a planet will result in a pressure minimum in the disk, and unless the dust and gas are extremely well coupled and the motion is governed by the gas (which will be true for sub-micron sized grains), the gas will respond to the pressure gradient while the dust will not. (The dust feels no viscous forces and much lower pressure forces than the gas and hence the tidal torques which create the gap will dominate.) If anything, the dust moves to regions of pressure maxima (see Haghighipour \& Boss 2003) on the edge of the gap. Furthermore, it is easier to form gaps in 2D disks than in 3D disks (see Kley 1999) and we expect the dust to settle to the midplane on a much shorter timescale than the gas phase. Both of these phenomena can occur at the same time. Thus predictions of observations of gap which assume a well-mixed disk are probably too pessimistic and it is therefore important to study the effects of a planet on the dust layer to help constrain the characteristics of the planets we might detect via the gaps they create.

While many semi-analytic and numerical studies have investigated the formation of planetary gaps in gas disks, very little work had been done on the effect of a planet in a dusty disk. Over the last few years, however, attention has begun to focus of the dust phase of 2D protoplanetary disks with embedded planets. Paardekooper \& Mellema $(2004,2006)$ studied the conditions for gap formation and showed that a smaller planetary mass is required to open a gap in the dust disk rather than in the gas. They note, however, that one should be careful when interpreting results for planets lighter than $0.1 M_{\text {Jup }}$ in two dimensional simulations. Rice et al. (2006) found that the pressure gradient at the outer gap edge can act as a filter keeping large grains out of the inner disk, which may explain the deficit of near-IR flux observed in the SED of some $\mathrm{T}$ Tauri stars. Alexander \& Armitage (2007) studied the dynamics of dust and gas during disk clearing and, adding an embedded planet, show that observations of transition disks can help discriminate between different models of disk clearing.

In this paper we study the formation of a gap triggered by an embedded planet in the dust layer of a protoplanetary disk. We will study the effects of planet mass and grain size on gap formation and evolution in 3D, two-phase (gas + dust) protoplanetary disks. These result will be compared with the gas-only simulations of others who must then assume the gas and dust are well mixed in order to determine how the dust-gas coupling (or decoupling) affects potential observations of planetary gaps.

\section{Gap opening criteria}

The gravitational perturbation of the planet launches a spiral density wave at the Lindblad resonances of the disk. The resulting tidal torques lead to an exchange in angular momentum when the waves damp by viscous dissipation or shocks. Waves exterior (interior) to the planet carry excess (deficit) angular momentum compared to the disk and hence when the wave damps, the disk gains (loses) angular momentum and moves outwards (inwards). This results in the formation of a gap around the planet.

In order to sustain the gap, there needs to be a balance between the tidal torques, which act to clear the gap, and the viscous torques, which act to fill the gap (Lin \& Papaloizou 1979). The gap criterion (Papaloizou \& Lin 1984; Bryden et al. 1999) is given by

$\begin{aligned} & \tau_{\text {tidal }}>\tau_{\text {visc }} \\ & \frac{M_{\mathrm{p}}}{M_{\star}}>40 \alpha_{\mathrm{SS}}\left(\frac{H}{r_{\mathrm{p}}}\right)^{2},\end{aligned}$

where $M_{\mathrm{p}}$ and $M_{\star}$ are the mass of the planet and star, $\alpha_{\mathrm{SS}}$ is the Shakura \& Sunyaev (1973) viscosity parameter, $H$ is the disk scale height and $r_{\mathrm{p}}$ is the semi-major axis of the planet. For inviscid disks, the competing forces are local pressure and gravitational tidal perturbations (Lin \& Papaloizou 1993) and gap criterion is given by

$$
\begin{aligned}
& F_{\text {tidal }}>F_{\text {press }} \\
& \frac{M_{\mathrm{p}}}{M_{\star}}>3\left(\frac{H}{r_{\mathrm{p}}}\right)^{3} .
\end{aligned}
$$

Our previous simulations (BF05) show that the thickness of the dust layer depends on the grain size because different sized grains fall to the midplane at different rates. The gas drag ensures that (for certain grain sizes which depend on the nebula conditions) the dust rapidly settles to the midplane and since the above expressions for the gap criteria depend on the disk scale height, this would suggest that it is easier to create and sustain a gap in the dust layer than in the gas. However, Eqs. (1) and (2) were derived without taking the drag force into account. Very small grains, which feel the strongest drag, are basically co-moving with the gas and are not likely to create a much sharper gap than the gas. Conversely very large grains (or boulders) are essentially decoupled from the gas and, feeling very little drag, have very long settling times. In this paper, we concentrate on the more interesting intermediate sized grains with the most efficient settling rates, for which the situation is greatly complicated by the drag forces and therefore numerical simulations are necessary to fully describe their behaviour. The regime we consider here is somewhat different to the $2 \mathrm{D}$ work of 
Paardekooper \& Mellema (2004) who study dusty disks in which the dust is strongly coupled to the gas and responds indirectly to the planet gravity through small radial pressure gradients in the gas disk that leads to the formation of a gap.

\section{Code description}

We have developed a 3D, two-fluid (gas+dust), locally isothermal, non-self-gravitating code based on the Smoothed Particles Hydrodynamics (SPH) algorithm, a Lagrangian technique described by Monaghan (1992). The dusty-gas is approximated by two inter-penetrating fluids that interact via aerodynamic drag. We assume Epstein drag, which is valid for the range of dust sizes and nebula parameters used in this study. Thus the drag force $F_{\mathrm{D}}$ is given by

$F_{\mathrm{D}}=\frac{4 \pi}{3} \rho_{\mathrm{g}} s^{2} v c$,

where $\rho_{\mathrm{g}}$ is the gas density, $s$ is the (spherical) grain radius, $c$ is the sound speed, and $v=\left|\boldsymbol{v}_{\mathrm{d}}-\boldsymbol{v}_{\mathrm{g}}\right|$ is the velocity difference between dust and gas, given by $\boldsymbol{v}_{\mathrm{d}}$ and $\boldsymbol{v}_{\mathrm{g}}$ respectively (Stepinski \& Valageas 1996). The dust particles are incompressible and there is no coagulation or evaporation of grains. All simulations in this paper are conducted with just one grain size at a time. In future work we shall include multiple grains sizes per simulation. For details of how the equations of motion and the density of the two fluids are calculated, we refer the reader to BF05.

\subsection{Implementation and initial conditions}

We set up a disk of gas and dust with a total mass of $M_{\text {disk }}$ around a one solar mass star into which we embed a planet of mass $M_{\mathrm{p}}$ at a distance of $r_{\mathrm{p}}$. The system is evolved for about 100 planetary orbits.

The code units are chosen such that $G=M_{\star}=r_{\mathrm{p}}=1$. The disk equation of state is isothermal and hence the temperature is constant in the vertical direction but follows a radial temperature profile $\left(T \propto r^{-1}\right)$. The isothermal sound speed at $r=1$ is $c=H / r=0.05$, where $H$ is the disk scale height and $r$ the radial distance from the star. Any heat produced in the disk due to tidal forces or viscous dissipation is assumed to be immediately radiated away. In order to compare our results to those of Paardekooper \& Mellema (2006), we use an initially constant surface density profile. In the $z$ direction particles are randomly distributed between $\pm H$. The intrinsic density of the dust grains, $\rho_{\mathrm{d}}$, is $1.25 \mathrm{~g} \mathrm{~cm}^{-3}$.

All simulations contain an equal number of gas and dust particles and the disks contain $1 \%$ dust by mass. We start our simulations with a gas disk containing 50000 particles and an embedded planet. This is evolved for 8 planetary orbits before we add the dust phase. The dust particles are initially overlaid on the gas particles and the system is then evolved for a further 96 orbits, making 104 orbits in total. Given that some accretion occurs during the first 8 orbits, we generally end up with about 96000 particles in total. While this may seem to be quite low resolution for today's SPH simulations (cf. $N_{\text {gas }} \sim 300000$ in Rice et al. 2003; and Schäfer et al. 2004) BF05 showed that this level of resolution gives the same results as simulations with almost twice as many particles (in the case of a disk without a planet). It should be noted that in our two-phase code, the time step is severely limited by the drag timescale. While the drag term is solved implicitly to alleviate the cripplingly short $\mathrm{d} t_{\mathrm{drag}}$ issue, this requires an extra $N / 2$ operations per time step (and an additional SPH particle neighbour search) and thus particle resolution suffers as a consequence. To ensure the validity of our low resolution results, we ran a series of high resolution simulations with $N \sim 400000$ which are presented in Sect. 4.1.

The planet is implemented as a point mass particle which moves under the gravitational influence of the central star, but does not feel pressure, viscous or drag forces. The SPH particles, on the other hand, feel the gravitational potential of the planet. The planetary orbit can either be circular, elliptic or even hyperbolic and is determined by the choice of the initial velocity. In this work we only study circular orbits. Particles are accreted by the planet if they cross a critical radius given by

$R_{\text {crit }}=0.1 R_{\text {Hill }}=r_{\mathrm{p}}\left(\frac{M_{\mathrm{p}}}{3 M_{\star}}\right)^{1 / 3}$.

We take no account of any mass or angular momentum transfer corresponding to this infall and therefore do not simulate migration of the planet. The central star also accretes particles that get closer than $r_{\text {in }}=0.4$ code units, which sets the inner edge of the disk. This rather large value was chosen to compare with the results of Paardekooper \& Mellema (2004, 2006). (See, however, Sect. 5.1 for a discussion about the location of the inner boundary and its effect on the gap.) Particles are also removed from the simulation if they escape to large radii, which is set to $r_{\mathrm{esc}}=4$ code units.

\subsection{Simulation suite}

In this work we present the results of a small, low mass disk close to the minimum mass solar nebula (MMSN). The disk mass is set to $2.9 \times 10^{-3} M_{\odot}$. Given the code scaling, $R_{\text {Hill }}=0.36 \mathrm{AU}$, the stellar accretion radius is $r_{\text {in }}=2 \mathrm{AU}$, and $r_{\mathrm{esc}}=20.8 \mathrm{AU}$, with the latter two setting the radial extent of the disk. Our standard model has a 1 Jupiter mass $\left(M_{\mathrm{Jup}}\right)$ planet on a circular orbit of radius 5.2 AU in a disk containing grains $1 \mathrm{~m}$ in size. Such a large grain size was chosen for our standard model because for these nebula conditions this is the grain size that settles most efficiently and thus the effect of the planet is most striking in a dust layer of $1 \mathrm{~m}$ sized "boulders". Further, boulders of this size should radially migrate most rapidly and here we will show that this migration can be stopped by planetary gaps. While the $1 \mathrm{~m}$ grains are observationally uninteresting, a large population of boulders would result in a population of smaller grains by collisions which are detectable.

We start by running our standard MMSN model and compare the evolution of the planetary gap in the gas and dust phases. We then run a series of experiments to study the effect of grain size in the dust disk, with $s=1 \mathrm{~cm}, 10 \mathrm{~cm}$ and $1 \mathrm{~m}$. This is followed by a series of experiments that study the effects of planetary mass on gap formation and evolution, with $M_{\mathrm{p}}=0.05,0.1,0.2,0.5$ and 1.0 $M_{\text {Jup }}$. The parameters of our simulation suite are summarised in Table 1 .

\subsection{Dust stopping time}

Since the drag force acting on the grains depends on the nebula gas density and relative velocity as well as the grain size, it is useful to know the stopping time for different grain sizes in order to compare with different nebula models. The stopping time (Stepinski \& Valageas 1996) in the Epstein drag regime (assuming solid spherical grains) is given by

$t_{\mathrm{s}}=\frac{m v}{F_{\mathrm{D}}}=\frac{\rho_{\mathrm{d}} s}{\rho_{\mathrm{g}} c}$ 
Table 1. Parameters of our simulation suite.

\begin{tabular}{llll}
\hline \hline \multicolumn{4}{c}{ MMSN models } \\
\hline Star mass & $1 M_{\odot}$ & Inner radius & $2 \mathrm{AU}$ \\
Disk mass & $2.9 \times 10^{-3} M_{\odot}$ & Outer radius & $20.8 \mathrm{AU}$ \\
\hline$s(\mathrm{~m})$ & $M_{\mathrm{p}}\left(M_{\text {Jup }}\right)$ & $\alpha, \beta$ & Drag \\
\hline 1.0 & 1.0 & $0.1,0$ & yes \\
\hline 0.1 & 1.0 & $0.1,0$ & yes \\
0.01 & 1.0 & $0.1,0$ & yes \\
\hline 1.0 & 0.5 & $0.1,0$ & yes \\
1.0 & 0.2 & $0.1,0$ & yes \\
1.0 & 0.1 & $0.1,0$ & yes \\
1.0 & 0.05 & $0.1,0$ & yes \\
\hline 1.0 & 1.0 & $0.1,0.5$ & yes \\
1.0 & 1.0 & 1,2 & yes \\
\hline 1.0 & 1.0 & $0.1,0$ & no \\
1.0 & 1.0 & $0.1,0$ & yes \\
\hline
\end{tabular}

where $\rho_{\mathrm{d}}$ is the intrinsic dust density. This is made nondimentional via $t_{\mathrm{s}}=T_{\mathrm{s}} / \Omega_{\mathrm{Kep}}$, and thus the non-dimensional stopping time, $T_{\mathrm{s}}$, can be written as

$T_{\mathrm{s}}=\frac{\rho_{\mathrm{d}} s \Omega_{\mathrm{Kep}}}{\rho_{\mathrm{g}} c}$

where $\Omega_{\text {Kep }}=v_{\text {Kep }} / r$ is the Keplerian angular velocity. We find that at the location of the planet for our standard model (see Sect. 4.1) the stopping time is given by $T_{\mathrm{s}}=0.067 \mathrm{~s}_{\mathrm{cm}}$ where $s_{\mathrm{cm}}$ is the grain size in $\mathrm{cm}$. Thus $T_{\mathrm{s}}=0.067,0.67$ and 6.7 for $1 \mathrm{~cm}$, $10 \mathrm{~cm}$ and $1 \mathrm{~m}$ grains respectively. This is in good agreement with Paardekooper \& Mellema (2006) (noting that they have an additional factor of $\sqrt{\pi / 8}$ in their expression of the stopping time, following Takeuchi \& Lin 2002).

\subsection{Viscosity}

Since protoplanetary disks are viscous accretion disks, we can apply the Shakura-Sunyaev $\alpha$-disk prescription (Shakura \& Sunyaev 1973) in which the kinematic viscosity is given by

$v=\alpha_{\mathrm{SS}} c H$.

In the thin disk approximation, $H / r=c / v_{\text {Kep }}$ and the viscosity can be rewritten as $v=\alpha_{\mathrm{SS}} c^{2} / \Omega$, and $\alpha_{\mathrm{SS}}$ is close to 0.01 (which provides a good fit to observations of protostellar disks - see Hartmann et al. 1998; King et al. 2007).

We use, as in BF05, the standard SPH artificial viscosity term (Monaghan \& Gingold 1983; Monaghan 1989) that is included in the SPH momentum equation and acts as a viscous "pressure". Using the SPH particle subscript notation $i$ and $j$, the viscous term of the momentum equation is given by

$\frac{\mathrm{d} v_{i}}{\mathrm{~d} t}=-\sum_{j} m_{j} \Pi_{i j} \nabla_{i} W_{i j}$,

where $v$ is the particle velocity, $m$ the particle mass, $W$ the smoothing kernel, and $\Pi$ the artificial viscosity term. The most common form of $\Pi_{i j}$ is due to Monaghan (1989) and is given by

$\Pi_{i j}=-\frac{\alpha \bar{c}_{i j} \mu_{i j}+\beta \mu_{i j}^{2}}{\bar{\rho}_{i j}}$,

where $\mu_{i j}=h \boldsymbol{v}_{i j} \cdot \boldsymbol{r}_{i j} /\left(r_{i j}^{2}+\eta^{2}\right)$ if $\boldsymbol{v}_{i j} \cdot \boldsymbol{r}_{i j}<0$ and is zero otherwise, $\bar{\rho}_{i j}$ and $\bar{c}_{i j}$ are the average density and average sound speed of interacting particles $i$ and $j$, and $\eta^{2}=0.01 h^{2}$ is a softening parameter that prevents $\mu_{i j}$ becoming singular, and $h$ is the SPH smoothing length. We use the cubic spline kernel of Monaghan \& Lattanzio (1985) given by

$W(\boldsymbol{r}, h)=\frac{\sigma}{h^{D}} \begin{cases}1-\frac{3}{2}(r / h)^{2}+\frac{3}{4}(r / h)^{3}, & \text { if } 0 \leq(r / h) \leq 1 \\ \frac{1}{4}(2-(r / h))^{3}, & \text { if } 1 \leq(r / h) \leq 2 \\ 0, & \text { otherwise, }\end{cases}$

where $D$ is the number of dimensions, and $\sigma$ is a normalisation constant given by $2 / 3,10 / 7 \pi$ and $1 / \pi$ in one, two and three dimensions respectively.

There are two free SPH viscosity parameters, $\alpha$ and $\beta$, which control the strength of the viscosity. (Note that the ShakuraSunyaev $\alpha_{\mathrm{SS}}$ and the SPH $\alpha$ are not the same.) The viscosity associated with the linear $\alpha$ term of $\Pi_{i j}$ was introduced to remove subsonic velocity oscillations that follow shocks (Monaghan \& Gingold 1983), while the nonlinear $\beta$ term damps high Mach number shocks and prevents particle interpenetration (Monaghan 1989). While it is reasonable to set $\beta$ to zero since we do not expect strong shocks in our protoplanetary disk simulations (cf. Bryden et al. 1999), some artificial bulk viscosity via the $\alpha$ term is needed to prevent random velocities from growing and producing a thick disk. Substituting Eqs. (9) and (10) into Eq. (8), it can be shown that $\alpha$ is related to the viscosity via

$v_{\mathrm{sph}}=\tilde{\sigma} \alpha c h$,

where $\tilde{\sigma}=1 / 8$ for a $2 \mathrm{D}$ cubic spline kernel or $2 / 15$ for a $3 \mathrm{D}$ cubic spline kernel (Pongracic 1988; Murray 1996). Equating Eqs. (7) and (11), we can relate $\alpha_{\mathrm{SS}}$ and $\alpha$ for our 3D simulations via

$\alpha_{\mathrm{SS}}=\alpha\left(\frac{\tilde{\sigma} h \Omega}{c}\right)$

In order to keep the corresponding Shakura-Sunyaev viscosity parameter $\alpha_{\text {SS }}$ close to 0.01 (as indicated by observations of protoplanetary disks - see Hartmann et al. 1998; King et al. 2007), we set the $\alpha=0.1$. A problem with using SPH to model planetary gaps is that the numerical viscosity cannot be reduced to arbitrarily small values, which means that (gas) particles will diffuse into the gap. Many numerical simulation of planetary gaps use grid-based codes (e.g. D'Angelo et al. 2002) in order to keep $\alpha_{\mathrm{SS}}$ as low as possible. Some grid codes can get $\alpha_{\mathrm{SS}}$ to be as low as $10^{-4}$. However, since observations of protoplanetary disks indicate $\alpha_{\mathrm{SS}} \sim 0.01$, SPH can do an adequate job and indeed one of the first simulations of an embedded planet in a disk by Sekiya et al. (1987) used SPH. Schäfer et al. (2004) present a new treatment of the viscosity in SPH codes which includes an additional artificial bulk viscosity term in the Navier-Stokes equation and demonstrate that their results compare well with grid-based codes. This is however very computationally expensive and has not been implemented in our code.

To check the validity of the SPH viscosity parameters, we ran a series of tests to investigate the effect of changing $\alpha$ and $\beta$. Our standard model has $\alpha=0.1$ and $\beta=0$, and we also ran test simulations with $(\alpha, \beta)=(0.1,0.5)$ and $(1,2)$. As expected, we find the $(\alpha, \beta)=(1,2)$ case to be much more dissipative than the two other. To quantify this, we calculate the percentage of particles lost between the 8th orbit (when the dust phase is incorporated) and 104th orbit (the end of the simulation). Particles can be lost either by accretion onto the central star, accretion onto the planet or escape to large radii. The results of these tests are given in Table 2 . We find that about twice as many particles are lost when $(\alpha, \beta)=(1,2)$ than in the other cases. Simulations with $(\alpha, \beta)=(0.1,0)$ and $(0.1,0.5)$ give similar results, with the latter being a little more dissipative. The evolution of the disk with 
Table 2. Percentage of particles lost due to accretion or ejection for various SPH viscosity parameters $\alpha$ and $\beta$.

\begin{tabular}{|c|c|c|}
\hline \multicolumn{2}{|c|}{ Viscosity parameters } & $\%$ of particles \\
\hline$\alpha$ & $\beta$ & lost \\
\hline 0.1 & 0 & $16.1 \%$ \\
\hline 0.1 & 0.5 & $16.6 \%$ \\
\hline 1 & 2 & $32.5 \%$ \\
\hline
\end{tabular}

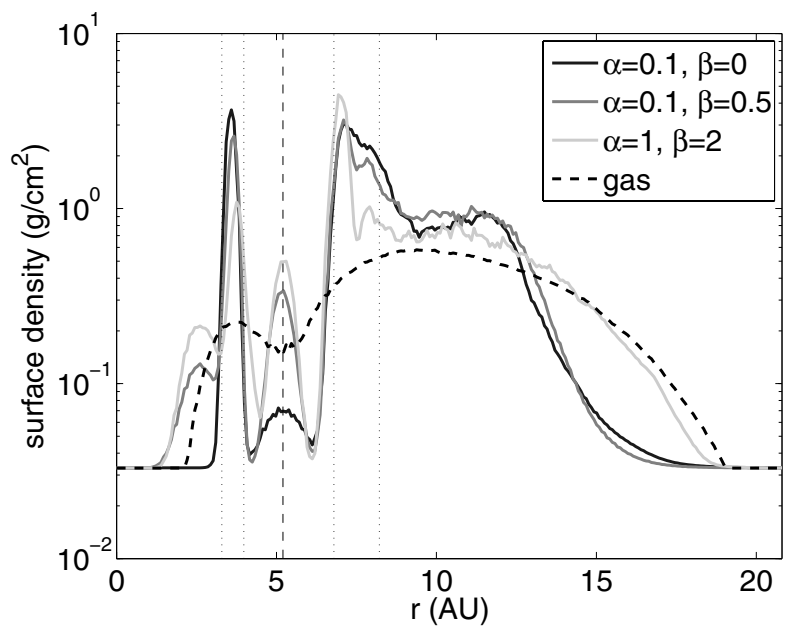

Fig. 1. Comparison of the dust disk surface density after 104 planet orbits for a range of SPH viscosity parameters, $\alpha$ and $\beta$, for our standard model with a $1 M_{\text {Jup }}$ planet at 5.2 AU. The gas surface density is scaled by a factor of 0.01 for direct comparison with the dust phase. The vertical lines are, from left to right, the 1:2 and 2:3 internal resonances, the 1:1 planetary orbit, and the $3: 2$ and 2:1 external resonances respectively.

different SPH viscosity parameters is shown in Fig. 1. We can see that the $(\alpha, \beta)=(1,2)$ simulations gives qualitatively different results, and that the $(\alpha, \beta)=(0.1,0)$ and $(0.1,0.5)$ simulations differ mainly in the corotation region and close to the edges of the gap where shock capture is more important.

In order to keep the viscosity as low as possible, and considering that results with $\beta=0$ are not so different from those with $\beta=0.5$, we keep $\alpha=0.1$ and $\beta=0$ for the remainder of our simulations.

\section{Simulation results}

Here we present the results from our suite of simulations, starting with our standard disk model, followed by our grain size tests and finally planetary mass tests.

\subsection{Standard model}

In the standard model, we set up a $2.9 \times 10^{-3} M_{\odot}$ disk containing $1 \%$ dust by mass made up of boulders $1 \mathrm{~m}$ in size. The disk extends from 2 AU to $20.8 \mathrm{AU}$ around a $1 M_{\odot}$ star and hosts a $1 M_{\text {Jup }}$ planet in a circular orbit at 5.2 AU.

In Fig. 2 we show the top-down $(x, y)$ and side-on $(r, z)$ view of the density structure in the gas and dust phases of the standard model. While the planet opens a shallow gap in the gas disk, the gap it creates in the dust layer is much more striking.

In Fig. 3 we plot the radial profile of the azimuthally averaged surface density of the standard model at five evolutionary stages $(8.4 P, 16 P, 32 P, 64 P$ and $104 P$, where $P$ is one

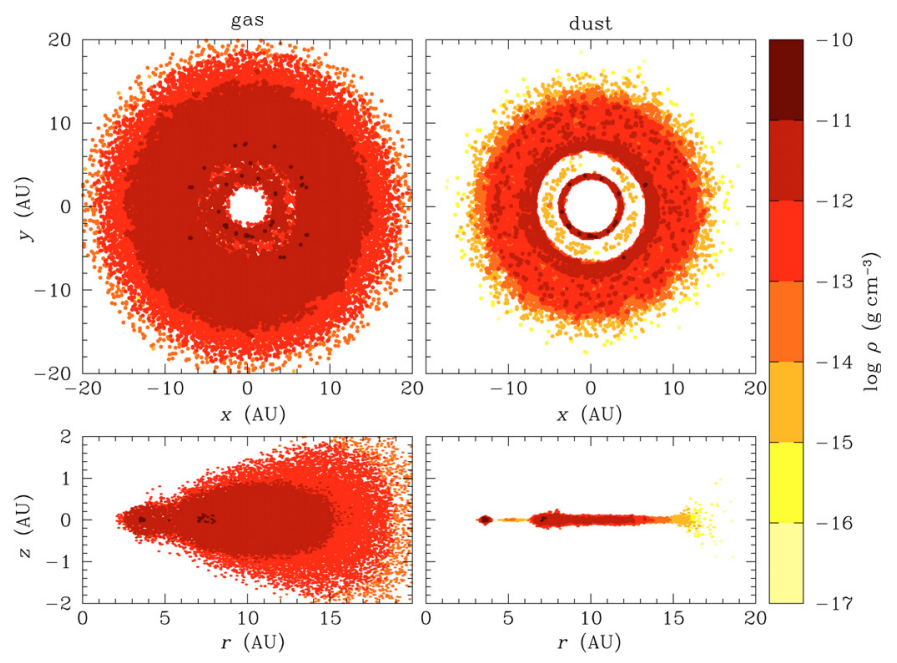

Fig. 2. End state of the standard model after 104 orbits (1230 years). Top: top-down view. Bottom: side-on view. Left: gas disk coloured by density. Right: dust disk coloured by density.

period of the planet). Note that the dip in the radial density profile due to the planet induced gap is surrounded by two density enhancements. The density enhancements are much sharper in the dust than in the gas even though the external enhancement gets broader over time due to feeding by external disk material. Particles that migrate inwards from the outer regions of the disk become trapped at the outer gap edge and over time more and more dust moves inwards and piles up at the outer gap edge, forcing this region of enhanced dust surface density to broaden. In the outer part of the disk, particles seem to be trapped by the 3:2 resonance as noted by Paardekooper \& Mellema (2004, 2006). We will return to this issue in Sect. 5.2. In the inner parts of the disk, on the other hand, particles flow through the resonances without becoming trapped due to the combined effects of the spiral waves created by the planet, the gas flow accreted by the central object, and the drag force that causes the dust to drift inwards faster than the gas once it has collapsed to the midplane. The internal over-density seen in Fig. 3 cannot be explained by an accumulation of inwardly migrating grains, since they cannot cross the gap. It is instead due to the accumulation of dust at the gas pressure maximum.

The gap is not exactly centred on the planet's orbit but it is shifted towards the inner disk. This phenomenon was also seen in the simulations of Paardekooper \& Mellema (2004) and is due to the fact that the dust does not directly feel the shock related to the creation of the gap. The shock only influences the dust via gas drag in the post-shock region. The flux of grains through the spiral wave is larger than if they were perfectly coupled to the gas and because the wave gradually depletes the gap, the density dip is deeper in the dust disk than in the gas.

Figure 4 shows the volume density of gas and dust as a function of distance to the star at the end of the simulation. The general radial dependence of the volume density is quite similar to that of the surface density seen in Fig. 3, with similarities seen in the shape of the dust profile around the gap and the accumulation of dust at the corotation radius. Indeed, dust particles tend to pile up where the gas concentrates, and Fig. 4 reveals a denser region of gas at the position of the planet which is harder to see in the vertically integrated surface density in Fig. 3, but which was hinted at by the reduced scale height of the gas disk in the lower left panel of Fig. 2. The most striking feature of Fig. 4 is that, because of the very efficient vertical settling, the dust 

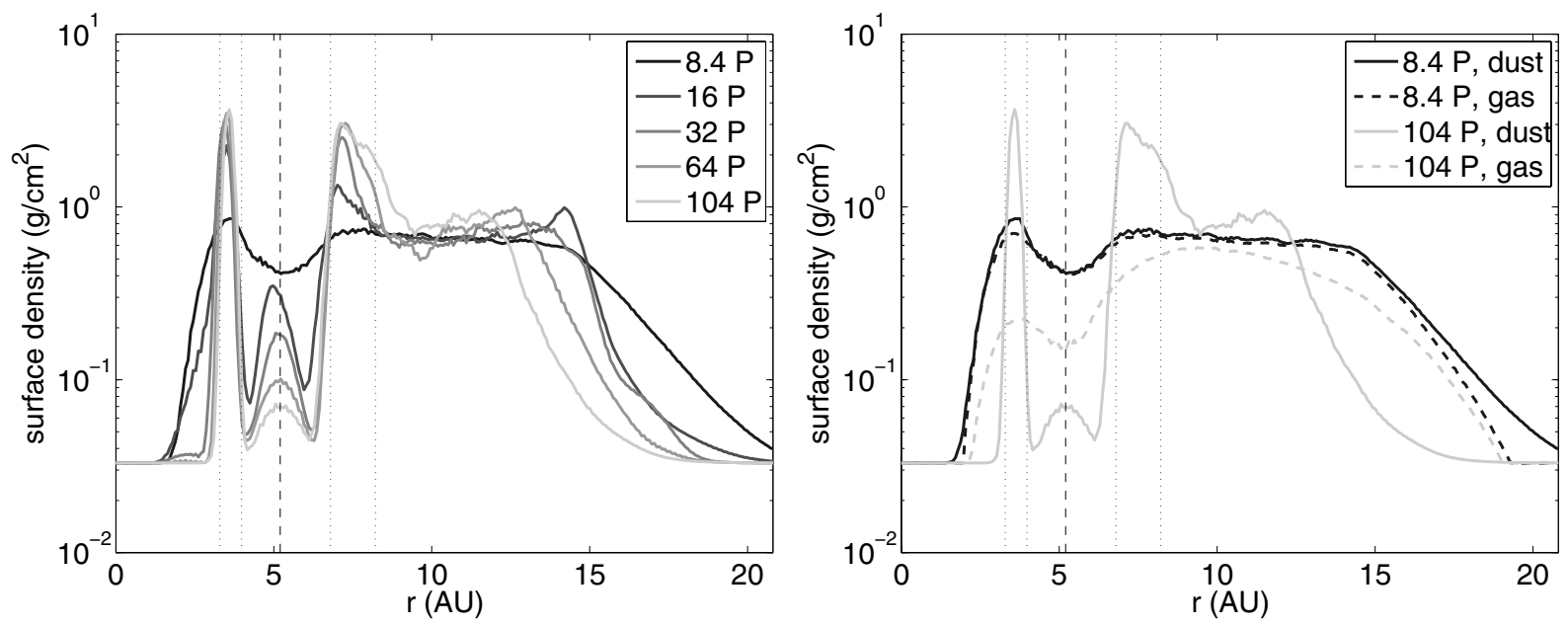

Fig. 3. Azimuthally averaged surface density profiles for a gap created by a $1 M_{\text {Jup }}$ planet at 5.2 AU in the standard model. The left panel shows the evolution of the $1 \mathrm{~m}$ sized grain dust layer after 8.4, 16, 32 and 64 and 104 planet orbits. The right panel shows the comparison of the gas and dust surface densities after 8.4 and 104 orbits. The gas density is scaled by 0.01 for direct comparison to the dust density. The vertical lines are the resonances as described in Fig. 1.

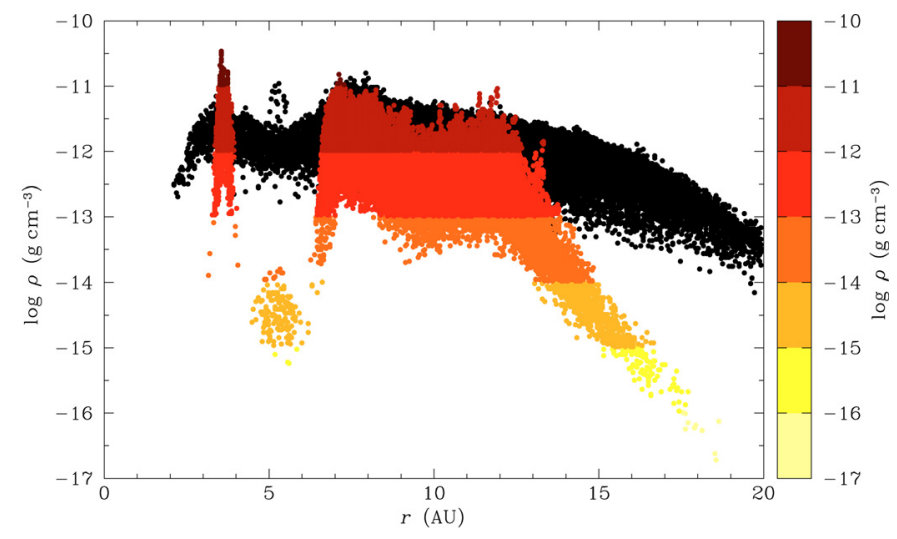

Fig. 4. Volume density profile of the gas and dust of the standard model after 104 orbits. The black points represent the gas particles and the coloured points represent the dust particles (coloured by density). Note that in this case the gas density has not been rescaled.

volume density reaches that of the gas in several regions of the disk, and even exceeds it at the inner edge of the gap where the dust piles up. Dust may therefore significantly affect the structure and evolution of the disk in that regime.

In order to better understand the resulting structures in the dust disk, we ran a high resolution $(N \sim 400000)$ simulation of our standard model and studied the particle loss rate. The simulations started with 200000 gas particles and after 8 orbits the 200000 dust particles were added. The particle numbers were compared at the end of the simulation. (Note that some gas particles are lost in the first eight orbits and hence we only compare particle numbers from the eighth orbit onwards.) In Table 3 we tally the number of gas and dust particles lost when $R>R_{\mathrm{esc}}$, when $R=R_{\text {crit }}$ (particles accreted by the planet), and when $R<R_{\text {inner }}$ (particles accreted by the star).

We find that about $12 \%$ of the gas particles are lost while less than $1 \%$ of the dust particles are lost. This is because dust particles tend to move inwards at the outer disk edge while gas particles tend to move outwards into the pressure void. At the edge of the planet gap, dust particles are trapped in the gas pressure maxima and so very few particles are actually captured by the planet. At the inner disk edge, the gas particles again feel the
Table 3. Particle loss rates from the 8th to the 104th orbit for high resolution runs of the standard MMSN model. Column one shows the accretion zone where particles are lost and columns two and three are the number of gas and dust particles lost respectively (with the percentage in brackets). Row one shows the total number of particles (gas + dust) remaining at $P=104$.

\begin{tabular}{|c|c|c|}
\hline Accretion & $N_{\mathrm{gas}}$ & $N_{\text {dust }}$ \\
\hline (Remaining) & \multicolumn{2}{|c|}{$329,841(87.5 \%)$} \\
\hline$R>R_{\mathrm{esc}}$ & $15663(4.2 \%)$ & $202(0.05 \%)$ \\
\hline$R=R_{\text {crit }}$ & $18746(5 \%)$ & $3,180(0.8 \%)$ \\
\hline$R<R_{\text {inner }}$ & $9424(2.5 \%)$ & $116(0.03 \%)$ \\
\hline
\end{tabular}

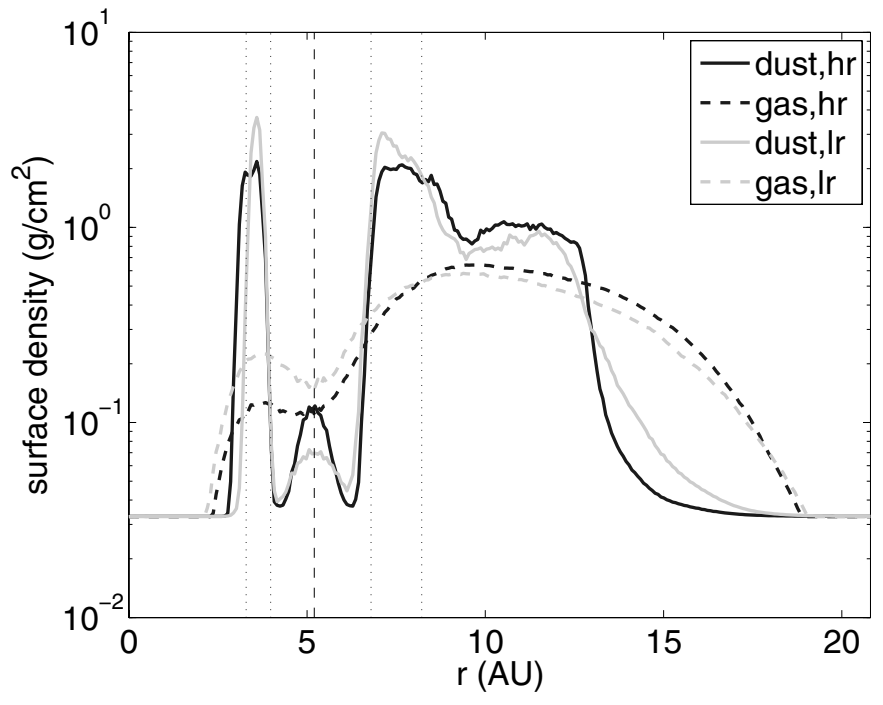

Fig. 5. The resulting gap for the standard model for the low resolution (lr: grey) and high resolution (hr: black) simulations. Dust profiles are shown as solid lines while gas profiles (scaled by 0.01 to compare with the dust) are shown as dashed lines.

pressure void and move inwards, while the dust particles remain where the gas pressure is highest.

Figure 5 compares the resulting gas and dust surface density profiles of the standard model for the low and high resolution simulations. The low resolution simulation captures the general 

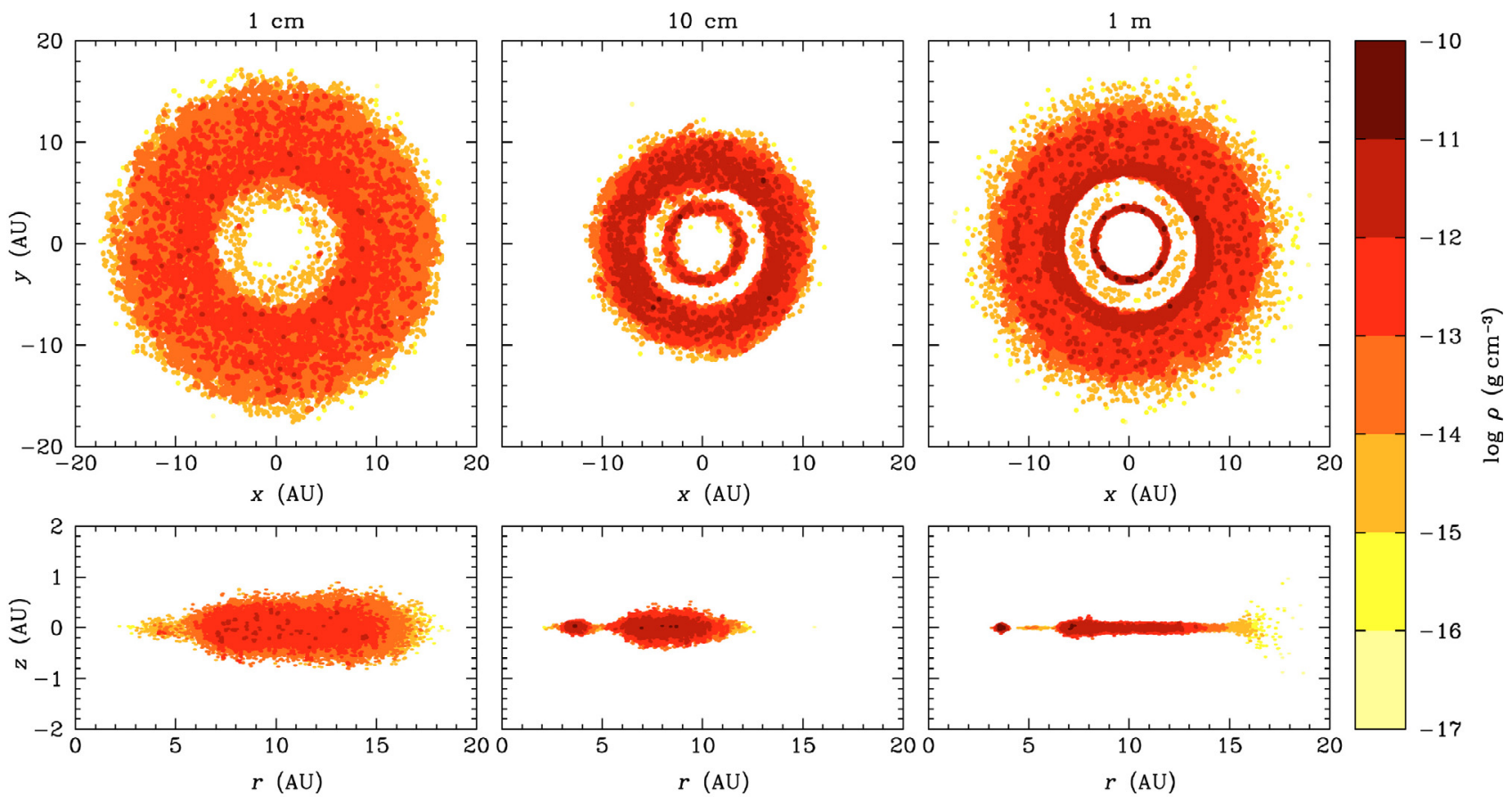

Fig. 6. Gap created in the dust disk for a range of grain sizes by a $1 M_{\text {Jup }}$ planet at $5.2 \mathrm{AU}$ after 104 orbits. Top panel shows the top-down view of disk and bottom panel shows the side-on view. From left to right: $1 \mathrm{~cm}, 10 \mathrm{~cm}$ and $1 \mathrm{~m}$ sized grains. The dust is coloured by density.

shape and depth of the gap quite well, though it slightly overestimates the peak density at the gap edges and underestimates the dust resonant trapping by the planet. We are confident that our low resolution simulations effectively capture the features of the planetary gap.

\subsection{Effect of grain size}

As discussed in Sect. 2, the scale height of the disk determines whether a planet of a given mass will open a gap in the disk, and from the results of BF05 we know that the evolution of the dust disk's scale height depends on the grain size. BF05 found that large bodies ( $s \geq 1 \mathrm{~m}$ ) are weakly coupled to the gas and essentially follow Keplerian orbits, while small bodies $(s \leq 10 \mu \mathrm{m})$ are very strongly coupled to the gas. In both cases, the dust disk remains flared but for different reasons. Only in the inner part of the disk, where the gas density is highest and hence the gas drag is most efficient, does the disk flatten in the case of large grains. For intermediate sized grains $(100 \mu \mathrm{m} \leq s \leq 10 \mathrm{~cm})$ BF05 found that the drag strongly affects the dust dynamics, resulting in rapid settling to the midplane and subsequent strong inward radial migration. They found that $10 \mathrm{~cm}$ grains experienced the fastest radial migration. While the nebula conditions of BF05 are not identical to those used in this study, we expect the results to be qualitatively similar.

We ran a series of simulations to determine the effect of grain size on the induced planetary gap. Three grain sizes are tested: $1 \mathrm{~cm}, 10 \mathrm{~cm}$ and $1 \mathrm{~m}$. Figures 6 and 7 show how the shape and depth of the gap in the dust phase of the MMSN disk varies according to the dust grains size. (Note that the gas disk is unaffected by a change in grain size and looks identical to the left hand panel of Fig. 2.)

Similar to BF05, we find that the radial migration is most efficient for the $10 \mathrm{~cm}$ sized grains over most of the disk, which differs from the findings of Weidenschilling (1977). This discrepancy can be explained by the different nebula parameters used, in particular the density. Indeed, Weidenschilling (1977) noted that the radial migration velocity reaches a maximum for grains which have $t_{\mathrm{s}} / t_{\mathrm{orb}} \simeq 1 / 2 \pi$, where $t_{\text {orb }}=2 \pi / \Omega_{\text {Kep }}$ is the orbital period. Such grains have therefore $T_{\mathrm{s}} \simeq 1$ and, following Eq. (6), one can estimate the "optimal" grain size for radial migration at a given location in the disk by

$s_{\mathrm{opt}}=\frac{\rho_{\mathrm{g}} c}{\rho_{\mathrm{d}} \Omega_{\mathrm{Kep}}}$.

Figure 8 displays the variation of $s_{\mathrm{opt}}$ with distance from the central star: decimetric grains are the most efficient radial migrators in the planet gap region, meter-sized grains migrate most efficiently from the outer gap edge out and decimetric grains take over again in the outer disk. Centimetre grains are only efficient at migrating in narrow regions on the very edges of the disk.

As a result, while the $1 \mathrm{~m}$ boulders have the most rapid settling rate, the $10 \mathrm{~cm}$ grains have the most rapid radial migration rate in the outer disk and produce a small, truncated, high density dust ring exterior to the planet. The density increase for the $10 \mathrm{~cm}$ grains in the external disk is comparable to that in the case of $1 \mathrm{~m}$ boulders due to two competing effects: the settling is a little less efficient and leads to a thicker dust layer than in the $10 \mathrm{~cm}$ dust simulations, but more dust coming from the outer edge, where the radial migration is more efficient, accumulates at the external edge of the gap. The $1 \mathrm{~cm}$ grains are most strongly coupled to the gas and thus their timescale of settling is much slower. This results in a broader, lower density dust disk. While the inner region of the $1 \mathrm{~cm}$-sized grain disk appears evacuated of dust (Fig. 6), this is due to the smaller density increase around the gap (Fig. 7) on the timescales shown. This is further supported by the lower accretion rate seen in the $1 \mathrm{~cm}$ grains than in the $10 \mathrm{~cm}$ grains.

As can be seen from Fig. 7, the gap gets deeper and wider as the grain size increases. Our findings are in contradiction to those of Paardekooper \& Mellema (2006) who claim that the grain size has no influence on the width of the gap (though they 


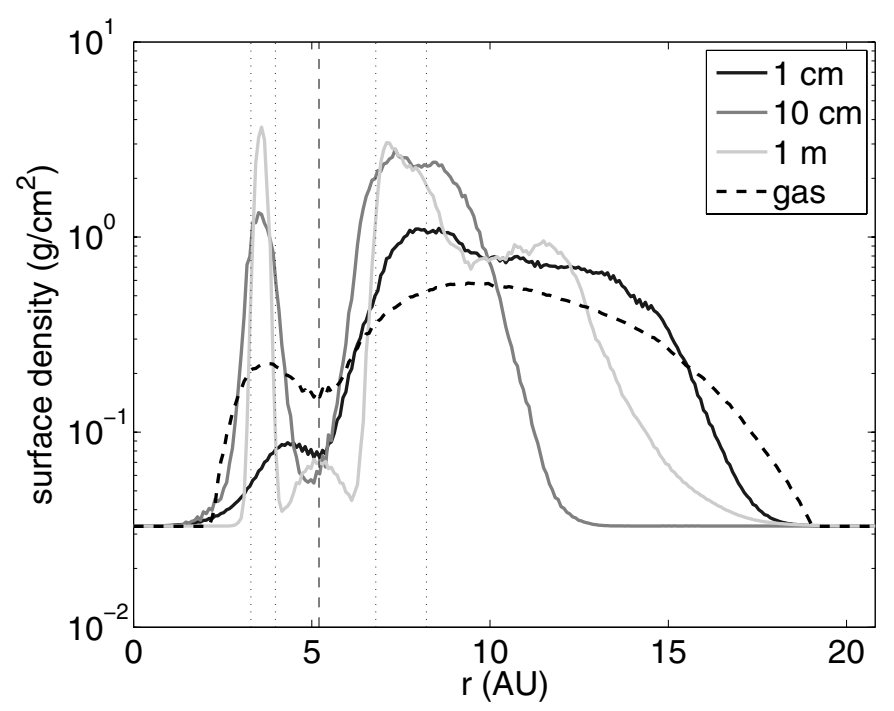

Fig. 7. Azimuthally averaged surface density profile for a gap created in the dust layer for different grain sizes $(1 \mathrm{~cm}, 10 \mathrm{~cm}, 1 \mathrm{~m})$ by a $1 M_{\text {Jup }}$ planet at $5.2 \mathrm{AU}$ after 104 orbits. Gas surface density is scaled by 0.01 to compare with the dust and the vertical lines show the resonances as in Fig. 1.

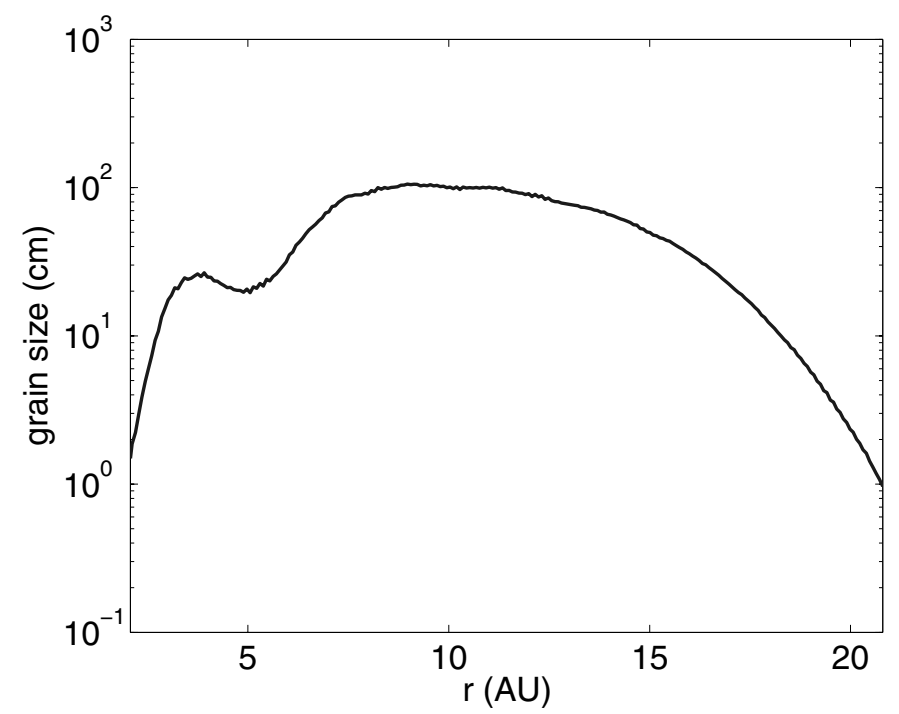

Fig. 8. Size of the grains with the largest radial migration velocity at a given radius in the disk.

do note that the gap gets deeper with increasing grain size as we do). While it is true that SPH will smooth the edges of the gap over $2 h$, we do not believe that this alone is the cause of the grain size dependent gap width. The $h$ values are about $0.1 \mathrm{AU}$ around the gap edges in the $1 \mathrm{~m}$ and $10 \mathrm{~cm}$ cases and 0.2 AU in the noisier $1 \mathrm{~cm}$ case, which is far smaller than the smoothing of the gap edges seen in Fig. 7. We assume, therefore, that this discrepancy is due to the evolution of the dust layer's scale height with grain size which we can follow in our 3D simulations. Within this size range, the dust disk scale height diminishes as the grain size increases, and hence the planet opens a gap more rapidly in larger grain sized dust layers.

For the $1 \mathrm{~m}$ boulders case, particles are found in corotation with the planet. We would expect that some particles would be found at corotation for all grain sizes, but we assume that this is only seen in the $1 \mathrm{~m}$ case because the gap is deeper and wider than in the other two cases, making those particles caught in corotation easier to see.

Rice et al. (2006) find that the pressure gradient at the outer gap edge act as a filter, allowing smaller particles to penetrate into the inner disk while holding back larger grains. Their transition size is $s \lesssim 10 \mu \mathrm{m}$ or smaller, and depends little on disk parameters. The dust grains we use in our simulations are all larger than this critical size and we indeed find that particles outside the gap do not migrate inwards, leaving the inner disk to drain by accretion on the central star.

\subsection{Effect of the planetary mass}

We also investigate the effect of the planetary mass on the evolution of the gap in the dust disk. We return to our standard MMSN model which contains $1 \mathrm{~m}$ sized grains and vary $M_{\mathrm{p}}$ from $0.05 M_{\text {Jup }}$ to $1 M_{\text {Jup }}$. The resulting disk morphologies are shown in Fig. 9. (Note that in this series of tests, the increasing planet mass does affect the gas disk distribution as well. However, the effect is quite small and so we do not include gas distribution figures.)

The azimuthally averaged surface density profiles after 104 orbits are shown in Fig. 10 for the dust and gas phases. An $0.05 M_{\text {Jup }}$ planet produces a decrease in the dust surface density in the vicinity of its orbit, whereas the gas surface density is not affected until the planet is more massive than $0.2 M_{\mathrm{Jup}}$.

As expected, the gap gets deeper and wider as the mass of the planet increases. In the inner disk, the dust surface density increase is due to the dust accumulation at the gas pressure maximum. While grains cannot cross the gap from the outer disk, in the inner disk grains move towards the pressure maximum coming from both sides of the maxima. The peak of the dust surface density is dictated by the efficiency of this process and does not depend on the gap depth. However the density peak is broader for smaller mass planets because of the correspondingly shallower gap. For low mass planets, material can still flow from the outer to inner disk and therefore we have more mass in the inner disk than when the gap is deep enough to prevent this inflow. In the case of larger mass planets, the density peak gets thinner before its maximal value starts to decrease.

In the outer disk, the maximum surface density increases a little with the mass of the planet but reaches almost the same value for the $0.2,0.5$ and $1 M_{\text {Jup }}$ planets. The peak is almost as broad in each case but is shifted to larger radii as the gap widens with increasing planetary mass.

For the two most massive planets modelled $\left(M_{\mathrm{p}}=0.5\right.$ and $1 M_{\text {Jup }}$ ) some dust particles appear to become trapped near corotation. Once again these are the simulations in which the gap is deepest and widest and hence it is easier to see particles in corotation.

In all cases but for the $1 M_{\text {Jup }}$ planet, the gap is again shifted slightly towards smaller radii than the planet orbit.

Paardekooper \& Mellema (2006) find that the smallest planet mass able to open a gap in a dust disk comprising $1 \mathrm{~mm}$-sized grains located at $13 \mathrm{AU}$ is $0.05 M_{\text {Jup }}$ for simulations of a MMSN disk. While their nebula parameters are not identical to ours and their models are 2D and less viscous ( $\left.\alpha_{\mathrm{SS}}=0.004\right)$, there is still general agreement between our results. From Eq. (1) we would expect a larger mass planet is required to open a gap at smaller radii, but with effective dust settling, the disk thickness is small at small radii which reduces the planet mass required to open a gap. Thus we also find that an $0.05 M_{\text {Jup }}$ planet at $5.2 \mathrm{AU}$ (for our disk parameters) can open a gap in the dust disk. 

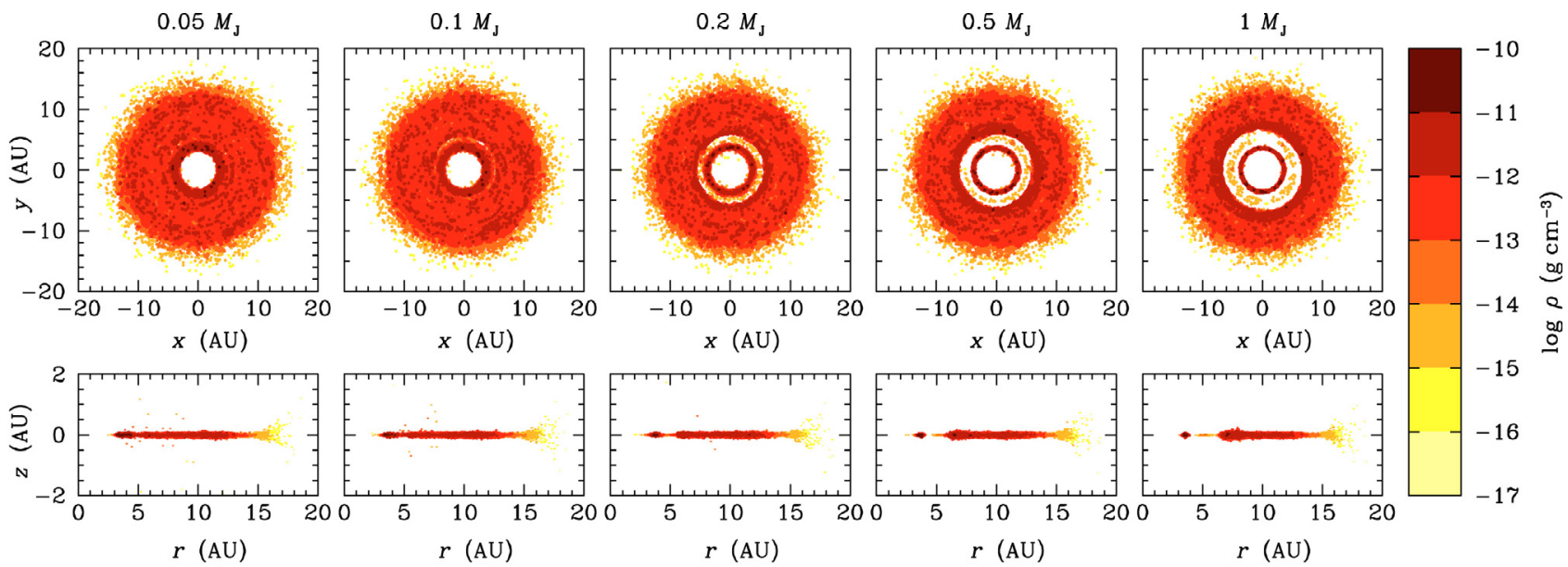

Fig. 9. Gap created in the dust disk comprising $1 \mathrm{~m}$ grains by a planet of varying mass at $5.2 \mathrm{AU}$ after 104 orbits. Top panel shows the top-down view of disk and bottom panel shows the side-on view. From left to right: a planet of mass $0.05,0.1,0.2,0.5$ and $1.0 M_{\text {Jup }}$ The dust is coloured by density.
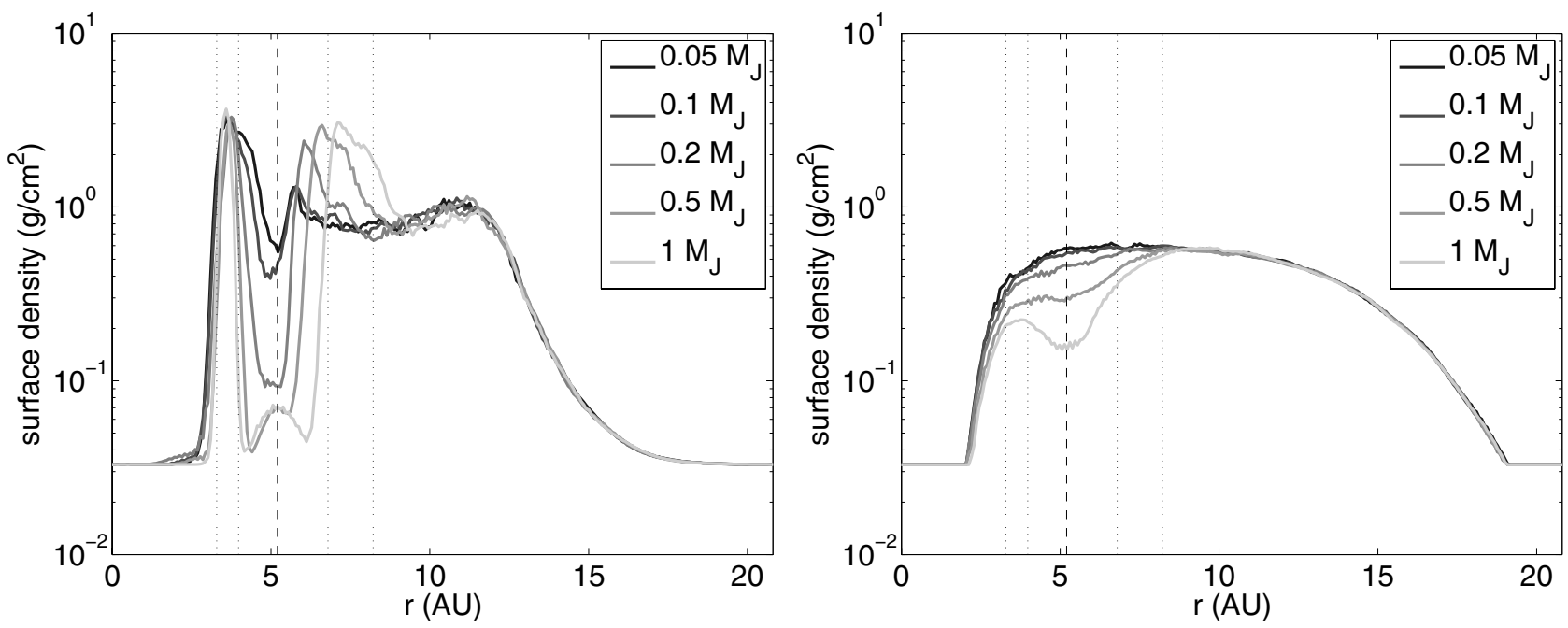

Fig. 10. Azimuthally averaged surface density profile for a gap created in the disk after 104 orbits by planets of various masses after 104 orbits. The left panel shows the evolution of the dust layer containing $1 \mathrm{~m}$ sized grains and the right panel shown the evolution of the gas layer which has been scaled by 0.01 for direct comparison with the dust. The vertical lines show the resonances as in Fig. 1 .

\section{Discussion}

Structures created by planets in dusty disks are more diverse than those created in the gaseous disks. With only aerodynamic drag, we already have the possibility of creating either a disk with a large central hole or a ring. Rice et al. (2006) also found that, for certain grain sizes, planets can create a central hole in the disk. Other processes can form similar structures, e.g. disk clearing by photoevaporation (Alexander \& Armitage 2007). The influence of radiation pressure and Poynting-Robertson drag would clearly lead to other more detailed structures for smaller grains (see, e.g. Takeuchi \& Artymowicz 2001).

\subsection{The effect of the inner boundary}

Recently Crida \& Morbidelli (2007) showed that the location of the inner boundary in planetary gap simulations can have a dramatic effect on the resulting structure of the innermost part of the disk. They found that a planet will open an inner cavity (i.e. an inner hole) in the disk if the planet is more massive that the disk or if the inner disk edge, $r_{\text {in }}$, is too close to the planet. They show that the disk surface density profile in the inner disk is lowered by a factor $1-\sqrt{r_{\text {in }} / r_{\mathrm{p}}}$ in the presence of a planet.

We re-ran our standard model with the inner boundary of the disk at $r_{\text {in }}=0.1$ and 0.25 to compare the resulting inner disk structure with our $r_{\text {in }}=0.4$ simulations. Using the formula of Crida \& Morbidelli (2007), we should find that the inner surface density drops by $32 \%$ when $r_{\text {in }}=0.1$, compared to a $63 \%$ decrease when $r_{\text {in }}=0.4$, which is what is seen in Fig. 11 (and compares well with Fig. 7 of Crida \& Morbidelli 2007).

Crida \& Morbidelli (2007) speculate about what would happen to dust in this inner disk region. They suggest that the dust would accumulate at the relative maximum of the gas density, which is indeed what we find, and that a thin dust ring would result, just as we see in our disks in Fig. 6.

While it is clear that the location of the inner boundary has a dramatic effect on the density distribution of the inner disk, the results we have presented for different grain sizes and different planet masses should not change for a fixed $r_{\text {in }}$. 


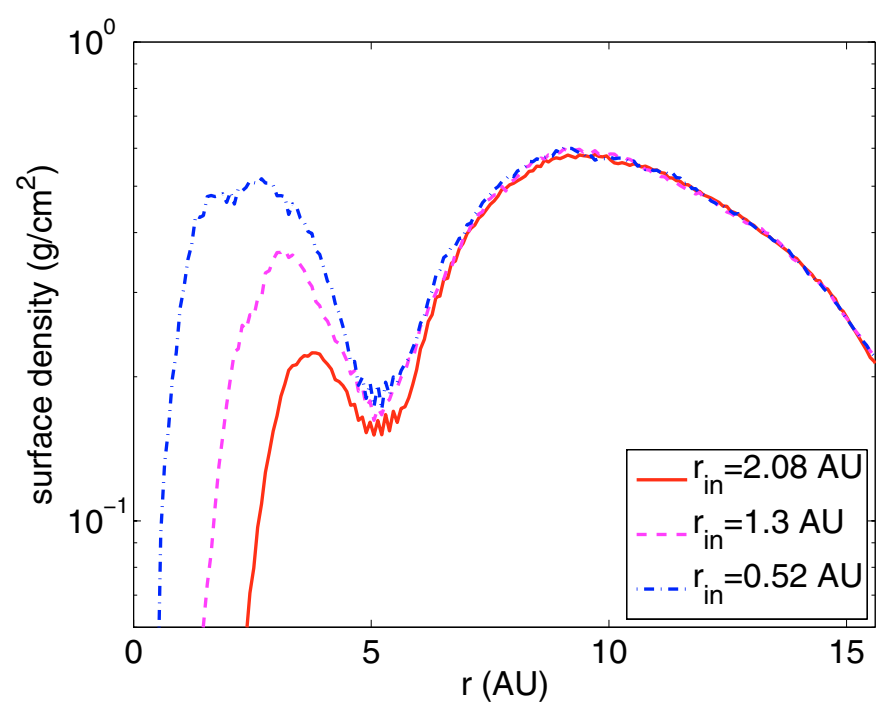

Fig. 11. Surface density profile of the gas in the standard model for three different inner boundaries: $r_{\text {in }}=2.08 \mathrm{AU}, 1.3 \mathrm{AU}$ and $0.52 \mathrm{AU}$.

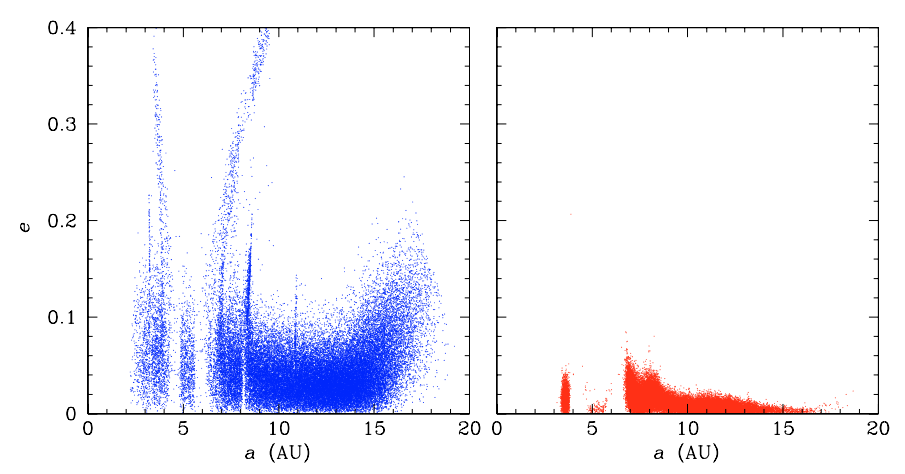

Fig. 12. Eccentricity $(e)$ versus semi-major axis $(a)$ for the standard MMSN disk with a $1 M_{\text {Jup }}$ planet orbiting at 5.2 AU after 104 orbits. Left panel: without gas drag. Right panel: gas drag included.

\subsection{Trapping in the $3: 2$ resonance}

Paardekooper \& Mellema (2006) suggested that particles become trapped in the 3:2 external resonance with the planet. While we see a clear density increase in the vicinity of the $3: 2$ resonance of our standard MMSN disk in Fig. 3, it appears to be too broad to caused by resonant trapping. To investigate further, we plot the eccentricity of the dust grains versus semi-major axis in Fig. 12.

In these kind of diagrams, resonances appear as thin vertical lines and as a V-shaped pattern at the edges of the gap. We ran a simulation without the drag force in which dust particles are fully decoupled from the gas. The results are shown on the left panel of Fig. 12 and the signatures of resonant trapping can be clearly seen. The rather high eccentricity of the dust particles is due to the initial conditions - the dust was initially given the velocity field of the gas and without drag there is no mechanism to damp the gas velocity dispersion. In the right panel of Fig. 12, we see that when simulations are run with gas drag, the drag is very efficient at damping the particles' eccentricity and consequently all resonant signatures disappear. Furthermore, if we compare our results for different grain sizes (see Fig. 7) and for different planet masses (Fig. 10), we see that the particle pile up at the outer edge of the gap does not always coincide with the $3: 2$ resonance for the same evolutionary phase (104 planet orbits). This seems to depend quite sensitively on the exact disk parameters.

We conclude, therefore, that the accumulation of dust that we and other authors (Paardekooper \& Mellema 2006; Alexander \& Armitage 2007) notice close to the outer gap edge is not due to resonant trapping. Resonant trapping will depend on the efficiency of the gas drag for the grain sizes under consideration.

\subsection{Observations of protoplanetary disks}

Our results have implications for recent predictions of observations of protoplanetary disks hosting planet gaps. As mentioned in Sect. 1, Wolf \& D'Angelo (2005) and Varnière et al. (2006) have used the results of 2D hydrodynamics simulations to produce synthetic images of protoplanetary disks following the suggestion of Paardekooper \& Mellema (2004) that such disks would be visible with ALMA. Such 2D simulations assume that the gas and dust are well mixed, which our results clearly demonstrate is not the case.

The results presented here cannot directly be used to construct synthetic observations because we only consider one discrete grain size per simulation. However, since we don't consider interactions between dust grains and since the structure of the gas disk doesn't vary with grain size, we can stack our results for different grain sizes to reconstruct a grain size distribution. With a finer size sampling than presented here, we will be able to create synthetic scattered light images (see Pinte et al. 2007).

Because our general findings show than the gap is generally more striking in the dust disk, we suggest that predictions of observations of protoplanetary disks are too pessimistic. Our results show that the density contrast around the gap can be very strong - up to four orders of magnitude (see Fig. 4) and this would be detectable. Our results, together with those of Paardekooper \& Mellema (2006), show that even if one varies the nebula parameters, the result is robust: an $0.05 M_{\text {Jup }}$ planet opens a gap in the dust disk. As suggested by Varnière et al. (2006) the resulting strong density enhancements at the edges of the gap should be easily visible with ALMA.

While the interactions of the dust with the gas disk and the planet are complex, our simulations can help constrain the planet mass and grain size distribution in future high angular resolution observations of protoplanetary disks.

\subsection{Enhanced planetesimal growth}

We have shown that the presence of the planet triggers an accumulation of grains at the external edge of the planetary gap, and the resulting density enhancement may well favour the growth of planetesimals in the region. This can be put in perspective with the results of Tsiganis et al. (2005), who propose a scenario for the formation of the Solar System that reproduces the characteristics of the giant planets as well as that of the Trojans and explain the late heavy bombardment of the Moon. This scenario relies on the controversial hypothesis that Jupiter and Saturn formed closer to one another than they are today and subsequently migrated, one inwards and the other outwards. While our results are rather extreme in that one of the planets is fully formed whereas the other is only at the planetesimal stage, even a planet embryo (well before it could carve out a gap) would create regions of pressure enhancement in its vicinity where dust would accumulate. Such dense rings would be ideal sites for the concentration of solid particles, and may lead to faster growth of planetesimals (Durisen et al. 2007). 


\subsection{Planet migration}

In this paper, we do not investigate the influence of planetary migration. The planet remains on a fixed circular orbit in our simulations and momentum exchange between planet and disk is not taken into account. We would expect, however, planet migration to have an effect on the dusty gap formation. Because the total dust mass is two orders of magnitude lower than the gas, one would expect the migration to be driven by gas and not dust. However, would this really be the case? Ignoring the influence of turbulence, once the dust has settled to the midplane its density tends to be comparable to that of the gas (see Fig. 4). One may speculate that the planet might be locked to the dust evolution and gas drag implies that dust flows faster towards the central object than gas. However, turbulence would need to be taken into account to fully study such a scenario. We will investigate the influence of migration in a dusty protoplanetary disk in a future paper.

\section{Conclusions}

We have conducted a series of 3D numerical simulations of twophase (dust + gas) protoplanetary disks to study the behaviour of the dust in the presence of a planet. We ran a series of experiments with a minimum mass solar nebula disk, varying the grain size and the planet mass.

We find that gap formation is more rapid and striking in the dust layer than in the gas layer. Varying the grain size alone results in a variety of different structures which may well be detectable with ALMA. For low mass planets in our MMSN disk, a gap was found to open in the dust layer while not in the gas layer.

Grains accumulate at the external edge of the gap but we find that this is not due to resonant trapping when gas drag is included. This is more likely due to the dust concentrating at the gas pressure maxima. Contrary to previous $2 \mathrm{D}$ work, our $3 \mathrm{D}$ simulations demonstrate that there is a dependence of the width and depth of the gap on grain size. Simulations like these can be used to help interpret observations to constrain the planet mass and grain sizes in protoplanetary disks.

Acknowledgements. Computations presented in this paper were performed at the Centre Informatique National de l'Enseignement Supérieur (France). We thank the Programme National de Physique Stellaire of CNRS/INSU, France, the Programme International de Cooperation Scientifique (PICS) France-Australia in Astrophysics (Formation and Evolution of Structures), and the Swinburne University Research Development Grant Scheme for partially supporting this research. We also thank the anonymous referee whose comments have greatly improved this paper.

\section{References}

Alexander, R., \& Armitage, P. J. 2007, MNRAS, 375, 500

Ardila, D. R., Golimowski, D. A., Krist, J. E., et al. 2004, ApJ, 617

Artymowicz, P. 2004, in Debris Disks and the Formation of Planets, ASP Conf. Ser., 39

Artymowicz, P., \& Lubow, S. H. 1996, ApJ, 467, L77

Balbus, S. A., \& Hawley, J. F. 1991, ApJ, 376, 214

Barrière-Fouchet, L., Gonzalez, J.-F., Murray, J. R., Humble, R. J., \& Maddison, S. T. 2005, A\&A, 443,185
Boss, A. P., \& Yorke, H. W. 1996, ApJ, 469, 366

Bryden, G., Chen, X., Lin, D. N. C., Nelson, R. P., \& Papaloizou, J. C. B. 1999, ApJ, 514, 344

Calvet, N., D’Alessio, P., Hartmann, L., et al. 2002, ApJ, 568, 1008

Crida, A., \& Morbidelli, A. 2007, MNRAS, 377, 1324

D'Angelo, G., Henning, T., \& Kley, W. 2002, A\&A, 385, 647

de Val-Borro, M., Edgar, R. G., Artymowicz, P., et al. 2006, MNRAS, 370, 529

Deller, A. T., \& Maddison, S. T. 2005, ApJ, 625, 398

Dullemond, C. P., \& Dominik, C. 2005, A\&A, 434, 971

Durisen, R. H., Boss, A. P., Mayer, L., et al. 2007, in Protostars and Planets V, ed. D. J. B. Reipurth, \& K. Keil (University of Arizona Press), 607

Fromang, S., \& Nelson, R. P. 2005, MNRAS, 364, 81

Fromang, S., \& Papaloizou, J. 2006, A\&A, 452, 751

Goldreich, P., \& Tremaine, S. 1979, ApJ, 233, 857

Goldreich, P., \& Tremaine, S. 1980, ApJ, 241, 425

Grigorieva, A., Artymowicz, P., \& Thébault, P. 2007, A\&A, 461

Haghighipour, N., \& Boss, A. P. 2003, ApJ, 598, 1301

Hartmann, L., Calvet, N., Gullbring, E., \& D’Alessio, P. 1998, ApJ, 495, 385

Johansen, A., \& Klahr, H. 2005, ApJ, 634, 1353

Johansen, A., Klahr, H., \& Henning, T. 2006, ApJ, 636, 1121

Jura, M., Malkan, M., White, R., et al. 1998, ApJ, 505, 897

Kalas, P., Graham, J. R., \& Clampin, M. 2005, Nature, 435, 1067

King, A. R., Pringle, J. E., \& Livio, M. 2007, MNRAS, 376, 1740

Kley, W. 1999, MNRAS, 303, 696

Lin, D. N. C., \& Papaloizou, J. 1979, MNRAS, 186, 799

Lin, D. N. C., \& Papaloizou, J. 1986, ApJ, 309, 846

Lin, D. N. C., \& Papaloizou, J. 1993, in Protostars and planets III, ed. E. H. Levy, \& J. I. Lunine (Tucson, AZ: Arizona Univ.), 749

Lufkin, G., Quinn, T., Wadsley, J., Stadel, J., \& Governato, F. 2004, MNRAS, 347,421

Maddison, S. T., Humble, R. J., \& Murray, J. R. 2003, in Scientific Frontiers in Research on Extrasolar Planets, ed. D. Deming, \& S. Seager, ASP Conf. Ser., 307

Masset, F. S., \& Papaloizou, J. 2003, ApJ, 588, 494

Monaghan, J. J. 1989, J. Comput. Phys., 82, 1

Monaghan, J. J. 1992, ARA\&A, 30, 543

Monaghan, J. J., \& Gingold, R. A. 1983, J. Comput. Phys., 52, 374

Murray, J. R. 1996, MNRAS, 279, 402

Ozernoy, L. M., Gorkavyi, N. N., Mather, J. C., \& Taidakova, T. A. 2000, ApJ, 537

Paardekooper, S.-J., \& Mellema, G. 2004, A\&A, 425, L9

Paardekooper, S.-J., \& Mellema, G. 2006, A\&A, 453, 1129

Papaloizou, J., \& Lin, D. N. C. 1984, ApJ, 285, 818

Pinte, C., Fouchet, L., Ménard, F., Gonzalez, J.-F., \& Duchêne, G. 2007, A\&A, 469, 963

Pongracic, H. 1988, Ph.D. Thesis, Monash University, Australia

Rice, W. K. M., Wood, K., Armitage, P. J., Whitney, B. A., \& Bjorkman, J. E. 2003, MNRAS, 342, 79

Rice, W. K. M., Lodato, G., Pringle, J. E., Armitage, P. J., \& Bonnell, I. A. 2004, MNRAS, 355, 543

Rice, W. K. M., Armitage, P. J., Wood, K., \& Lodato, G. 2006, MNRAS, 373, 1619

Schäfer, C., Speith, R., Hipp, M., \& Kley, W. 2004, A\&A, 418, 325

Schneider, G., Smith, B. A., Becklin, E. E., et al. 1999, ApJ, 513

Schneider, G., Silverstone, M. D., Hines, D. C., et al. 2006, ApJ, 650, 414

Sekiya, M., Miyama, S. M., \& Hayashi, C. 1987, Earth, Moon and Planets, 39, 1

Shakura, N. I., \& Sunyaev, R. A. 1973, A\&A, 24, 337

Stepinski, T. F., \& Valageas, P. 1996, A\&A, 309, 301

Takeuchi, T., \& Artymowicz, P. 2001, ApJ, 557, 990

Takeuchi, T., \& Lin, D. N. C. 2002, ApJ, 581, 1443

Takeuchi, T., Miyama, S. M., \& Lin, D. N. C. 1996, ApJ, 460, 832

Tsiganis, K., Gomes, R., Morbidelli, A., \& Levison, H. F. 2005, Nature, 435, 459

Varnière, P., Bjorkman, J. E., Frank, A., et al. 2006, ApJ, 637, L125

Ward, W. R. 1997, Icarus, 126, 261

Weidenschilling, S. 1977, MNRAS, 180, 57

Weinberger, A. J., Becklin, E. E., Schneider, G., et al. 1999, ApJ, 525, 53

Wilner, D. J., Holman, M. J., Kuchner, M. J., \& Ho, P. T. P. 2002, ApJ, 569

Wolf, S., \& D'Angelo, G. 2005, ApJ, 619, 1114

Wyatt, M. C. 2006, ApJ, 639, 1153 